Original Research Paper

\title{
Warranty Cost Models using Accelerated Life Tests on Dagum Distribution
}

\author{
Eman A. El-Dessouky Nassef \\ Department of Statistics, Faculty of Commerce, Al- Azhar University, Egypt
}

\author{
Article history \\ Received: $10-05-2020$ \\ Revised: 06-06-2020 \\ Accepted: 25-07-2020 \\ Email: emdessouky@hotmail.com \\ emaneldessouky501.el@azhar.edu.eg
}

\begin{abstract}
Warranty one of the main factors which effect on the decision to purchase any product whether it is non repairable or repairable, therefore, in market the manufacturers can compete by using warranty service. The cost of warranty are wanted to predict which are mirrored on the price and profitability of products. To achieve this goal, the research is concerned with predicting the cost of the two common types of warranty models which are free rebate warranty and pro-rata rebate warranty when The lifetime of items is assumed to follow Dagum distribution. The constant stress partially accelerated life tests based on type II censoring is used. Maximum likelihood method is used to estimate the model parameters and acceleration factor of lifetime distribution from the test data. Confidence Interval for the model parameters are constructed using normal approximation and bootstrap method. Finally, Some numerical illustrations are provided.
\end{abstract}

Keywords: Constant-Stress, Partially Accelerated Life Test, Accelerated Factor, Type II Censoring, Bootstrap, Free Rebate Warranty, Pro-Rata Rebate Warranty

\section{Introduction}

According to competitive markets, products are bought with warranty. Therefore, manufacturer need to predict the price of claims due to failures in the course of a specific period, which known as warranty length. These costs are fluctuated due to many factors such as: Probability density characteristic of failure time distribution, duration of warranty period, type of warranty policy, average cost of replacement or repair. Warranty cost will be reflected in the sale price considering it as indirect costs and how to determine the warranty cost per unit. The determination of warranty costs will help manufacturers plan operations more effectively since an accurate knowledge of warranty costs allows more accurate profit expectations which may lead to unanticipated marketing advantages.

Numerous warranty cost models are developed to predict it as (Wang, 2006; Rahman, 2007; Amberkar and Jagtap, 2014; Chen et al., 2017). Also, some of them used Accelerated life testing in predicting warranty cost models as: Yang (2010; Zhao and Xie, 2017), when life time distribution is Weibull distribution.

Accelerated Life Testing (ALT) is a method for estimating the reliability of products at normal operating conditions from the failure data obtained at the severe conditions. Accelerated life testing methods are also useful for obtaining information on the life of products or materials over a range of conditions, which are encountered in practice. Some information can be obtained by testing over the range of conditions of interest or over more severe conditions and then extrapolating the results over the range of interest, (Sen, 1999). Partially Accelerated Life Testing (PALT) a approach of life testing is proposed which combines each normal and accelerated life-testing procedures. It is assumed that an item can be tested either in a standard environment or under stress. The amount of stress is fixed in advance and is the same for all items to be tested (Mittal, 2013). For an overview of constant-stress PALT, there is amount of literature on designing PALT for probability distributions: Bai and Chung (1992; Degroot and Goel, 2006) for the Exponential distribution. Bai et al. (1993) for the lognormal distribution, (Ismail, 2014) for Pareto lifetime distribution, (Ismail, 2009) for Weibull distribution, (Abdel-Ghaly et al., 2008; Cheng and Wang, 2012) for Burr type-XII distribution, (Zarrin et al., 2012) for Rayleigh distribution, finally, (Kamal et al., 2013), for Inverted Weibull distribution.

The test procedure of the constant stress PALT and its assumptions are described as follows: 


\section{Test Procedure}

In a constant stress PALT, the total sample size $n$ of test units is divided into two parts such that:

1. $n \pi$ items randomly chosen among $n$ test items sampled are allocated to accelerated condition and the remaining are allocated to normal use condition, where $\pi$ is the proportion of test items

2. Each test item is run until the occurrence of $r_{u}, r_{a}$ number of failures and the test condition is not changed

\section{Assumptions}

1. The lifetimes $T i, i=1, \ldots, n(1-\pi)$ of items allocated to normal use condition, are i.i.d. r.v.'s

2. The lifetimes $X j, j=1, \ldots, n \pi$ of items allocated to accelerated condition, are i.i.d r.v.'s

3. The lifetimes $T i$ and $X j$ are mutually statisticallyindependent

The rest of this paper is organized as follows: In section 2 the Dagum distribution is introduced as a failure time model. The warranty cost models are described in section 3. Section 4 presents the maximum likelihood estimates of the model parameters. The confidence interval for model parameters are constructed by using normal approximation method and boot strap method in section 5. To illustrate the theoretical results, simulation studies are carried out in section 6. Finally, section 6 contains the conclusions.

\section{The Model}

This section introduces the assumed model for product life.

\section{Notations}

$T \quad$ lifetime of an item at normal use condition

$X \quad$ lifetime of an item at accelerated use condition

$\lambda \quad$ acceleration factor $\lambda>1$

$\alpha, \beta \quad$ the shape parameters of the Dagum distribution

$\theta$ the scale parameter of the Dagum distribution

$t_{i} \quad$ Observed life time of item $i$ tested at normal condition

$x_{j} \quad$ Observed life time of item $j$ tested at accelerated condition

$n \quad$ Sample size (total items to be tested in PALT)

$n_{u}, n_{a} \quad$ Number of test items at normal and accelerated condition respectively.

$r_{u}, r_{a} \quad$ Number of censoring items at normal and accelerated condition respectively.

$n_{s u}, n_{s a} \quad$ Number of survival items at normal and accelerated condition respectively.

$\pi \quad$ The proportion of test items.

$t_{w f} \quad$ Period of free rebate warranty $t_{w p} \quad$ Period of pro-rata rebate warranty

$c_{w} \quad$ Average of warranty cost per unit

PALT Partially accelerated life test

CSPALT Constant Stress Partially accelerated life test

FRW Free rebate warranty

PRW Pro-rata Rebate Warranty

\section{Failure Time Distribution as Dagum Distribution}

The lifetimes of the test items are assumed to follow a three -parameter Dagum distribution. This distribution was first introduced by (Dagum, 1977) as a new model of personal income-distribution-specification. Recently many studies are interested in modeling lifetime data or survival data with Dagum distribution as.

Domma et al. (2011), showed that the Dagum distribution may be a competitive model for describing data which include censored observations in lifetime and reliability problems and estimated its parameters using maximum likelihood. Domma et al. (2012), used dagum distribution in reliability analysis. Al-Zahrani (2016), used dagum distribution as an effective probability distribution that can be considered for studying the lifetime of a product/material and proposed it for reliability test plans. Domma et al. (2018), used Dagum distribution as a model of income inequality and poverty measures.

So, it is a statistical distribution frequently used in life data analysis. The probability density function (pdf) of three-parameter Dagum distribution is given by:

$$
f(t)=\alpha \beta \theta t^{-(\alpha+1)}\left(1+\theta t^{-\alpha}\right)^{-(\beta+1)} \quad ; t \geq 0, \alpha, \beta, \theta>0
$$

The cumulative function is given by:

$$
F(t)=\left(1+\theta t^{-\alpha}\right)^{-\beta} \quad ; t \geq 0, \alpha, \beta, \theta>0
$$

The reliability function is:

$$
R(t)=1-\left(1+\theta t^{-\alpha}\right)^{-\beta} \quad ; t \geq 0, \alpha, \beta, \theta>0
$$

The hazard function is:

$$
h(t)=\frac{\alpha \beta \theta t^{-(\alpha+1)}\left(1+\theta t^{-\alpha}\right)^{-(\beta+1)}}{1-\left(1+\theta t^{-\alpha}\right)^{-\beta}} \quad ; t \geq 0, \alpha, \beta, \theta>0
$$

\section{Warranty Cost Models}

There are many different types of warranty models such as: Free Warranty, Pro-Rata Warranty, Lump -Sum Rebate Warranty. These policies may be renewable or ordinary (Non-renewable). Also, it may be combined between two or more warranty model as the combination of the free warranty model followed by the pro-rata 
warranty model. We can distinguish between the non repairable products and the repairable products, when designing the warranty policies.

Many researchers are interested in warranty cost models as: Murthy and Rodin (1990), developed a new model for item usage and item failure and obtained the expected cost of servicing warranty per unit when the product is sold with warranty. Díaz et al. (2009), reviewed a list of important models for warranty costs, following a chronological tour and describing these studies in a general way to the framework of warranty cost management. Park and Pham (2010), presented warranty cost models are based on the quasi-renewal processes and exponential distribution. Amberkar and Jagtap (2014) dealt with models that may be used to obtain these cost estimates for products sold with freereplacement and pro rata warranties. Chen et al. (2017), developed warranty cost modeling, the effects of various parameters, such as the burn-in time and warranty Length Optimization Under two types of failure and combination free replacement and pro-Rata warranty.

We will deal with the most two common warranty policies used: FRW and PRW.

In the warranty period, the failures of the system or product can be modeled as non homogeneous Poisson process (NHPP) with an intensity function $h(t)$ (Blischke and Murthy, 1994). Therefore the expected number of failures, the expected warranty cost per unit and the expected of total warranty cost during the warranty period are obtained as follows:

\section{In Free Rebate Warranty (FRW)}

Where the period of warranty is $t_{w f}$, the expected number of failures is:

$$
E\left(N_{w f}\left(t_{w f}\right)\right)=\int_{0}^{t_{w f}} h(t) d t
$$

where, $h(t)$ is the hazard rate function and if modelled as Dagum distribution as given in Equation (4), then:

$$
\begin{aligned}
& E\left(N_{w f}\left(t_{w f}\right) \mid \alpha, \beta, \theta\right)=\int_{0}^{t_{w f}} \frac{\alpha \beta \theta t^{-(\alpha+1)}\left(1+\theta t^{-\alpha}\right)^{-(\beta+1)}}{1-\left(1+\theta t^{-\alpha}\right)^{-\beta}} d t \\
& =-\ln \left(1-\left(1+\theta t_{w f}^{-\alpha}\right)^{-\beta}\right)
\end{aligned}
$$

Under FRW if an item fails before the end of warranty length, it is replaced or repaired at no cost to the customer and the replaced or repaired item has a warranty.

Therefore, the expected warranty cost per unit is given by:

$$
\begin{aligned}
& \left.C_{w f}\left(t_{w f}\right) \mid \alpha, \beta, \theta\right)=C_{w} \int_{0}^{t_{w f}} f(t) d t \\
& =C_{w}\left(1+\theta t_{w f}^{-\alpha}\right)^{-\beta}
\end{aligned}
$$

The prediction of total warranty cost for FRW model is:

$$
\begin{aligned}
& E\left(C N_{w f}\left(t_{w f}\right) \mid \alpha, \beta, \theta\right) \\
& =-\ln \left(1-\left(1+\theta t_{w f}^{-\alpha}\right)^{-\beta}\right)\left(C_{w}\left(1+\theta t_{w f}^{-\alpha}\right)^{-\beta}\right)
\end{aligned}
$$

\section{In Pro-Rata Rebate Warranty (PRW)}

Where the period of warranty is $t_{w f}$, the expected number of failures is $E\left(N_{w p}\left(t_{w p}\right)\right)=\int_{0}^{t_{w p}} h(t) d t$, then:

$$
E\left(N_{w p}\left(t_{w p}\right) \mid \alpha, \beta, \theta\right)=-\ln \left(1-\left(1+\theta t_{w p}^{-\alpha}\right)^{-\beta}\right)
$$

Under PRW if an item fails before the end of warranty length, it is replaced or repaired at a cost that depend on the age of the item at the time of failure and the replacement or repaired item is covered by an identical warranty. A discount proportional to the remaining length of the warranty is given on the purchase price of the replacement or repaired item.

Therefore, the expected warranty cost per unit is given by:

$$
\begin{aligned}
& \left.C_{w p}\left(t_{w p}\right) \mid \alpha, \beta, \theta\right)=C_{w} \int_{0}^{t_{w p}}\left(1-\frac{t}{t_{w p}}\right) f(t) d t \\
& =C_{w}\left(\left(1+\theta t_{w p}^{-\alpha}\right)^{-\beta}\right)-\beta \theta^{\frac{1}{\alpha}} I B\left(\Upsilon_{1}, \Upsilon_{2}, \theta t_{w p}^{-\alpha}\right)
\end{aligned}
$$

where, $I B\left(\Upsilon_{1}, \Upsilon_{2}, \theta t_{w p}^{-\alpha}\right)$ denotes to incomplete Beta function:

$$
\Upsilon_{1}=1-(1 / \alpha), \Upsilon_{2}=\beta+(1 / \alpha)
$$

The prediction of total warranty cost for PRW model is:

$$
\begin{aligned}
& \left.E\left(C N_{w p}\left(t_{w p}\right) \mid \alpha, \beta, \theta\right)\right) \\
& \left.=-\ln \left(1-\left(1+\theta t_{w p}^{-\alpha}\right)^{-\beta}\right)\left(C_{w p}\left(t_{w p}\right) \mid \alpha, \beta, \theta\right)\right)
\end{aligned}
$$

Therefore, we need to estimate the value of parameters $\alpha, \beta$ and $\theta$ to predicate the expected warranty cost under FRW and PRW.

\section{Maximum Likelihood Parameter Estimation}

Maximum likelihood estimation is used with a wide range of statistical analyses for its properties. So, we can depend on it to estimate the acceleration model parameters at the same time as life distribution parameters.

However, in a simple constant stress PALT the time $T$ at which an item on test is taken out of the normal use 
condition and put part of sample size items under stress. When an item is put under stress its lifetime is changed by the factor $\lambda$ Let the random variable $T$ denote the lifetime of an item in the normal use condition and let $x$ denote its lifetime under the partially accelerated test procedure just described.

Since the lifetimes of the test items follow the Dagum distribution, the probability density function of an item tested at normal use condition is given as in (1). While for an item tested at accelerated use condition, the pdf is given by:

$$
\begin{aligned}
& f(t)=\alpha \beta \theta \lambda(\lambda x)^{-(\alpha+1)}\left(1+\theta(\lambda x)^{-\alpha}\right)^{-(\beta+1)} \\
& ; x \geq 0, \alpha, \beta, \theta>0, \lambda>1
\end{aligned}
$$

where, $x=\lambda^{-1} T$.

Since the test in type-II censoring terminates when a predetermined total number of failures $r_{u}$ and $r_{a}$ are reached, so, the observed lifetimes $t_{(1)}<t_{(2)}<\cdots<t_{(r u)}$ and $x_{(1)}<x_{(2)}<\cdots<x_{(r a)}$ are ordered failure times at normal use and accelerated conditions respectively, where $t_{r_{u}}$ and $x_{r_{a}}$ is the time of the $r$ th failure at which the experiment is terminated, $r_{u}$ and $r_{a}$ are the numbers of items failed at normal use and accelerated use conditions, respectively.

Let the indicator functions: $\delta_{u i}=I\left(T_{i} \leq t_{r u}\right)$ and $\delta_{a j}=$ $I\left(X_{i} \leq x_{r a}\right)$.

Then the likelihood for $\left(t_{i}, \delta_{u i}\right)$, the likelihood for $\left(x_{j}\right.$, $\left.\delta_{a j}\right)$ and the total likelihood for are respectively given by:

$$
\begin{aligned}
& L_{u i}\left(t_{i}, \delta_{u i} \mid \alpha, \beta, \theta\right) \\
& =\prod_{i=1}^{n(1-\pi)}\left[\alpha \beta \theta t_{i}^{-(\alpha+1)}\left(1+\theta t_{i}^{-\alpha}\right)^{-(\beta+1)}\right]^{\delta_{u i}}\left[1-\left(1+\theta t_{r u}^{-\alpha}\right)^{-\beta}\right]^{1-\delta_{u i}}
\end{aligned}
$$

The likelihood function for $\left(x_{j}, \delta_{a j}\right)$ is given by:

$$
\begin{aligned}
& L_{a j}\left(x_{j}, \delta_{a j} \mid \alpha, \beta, \theta, \lambda\right) \\
& =\prod_{j=1}^{n \pi}\left[\alpha \beta \theta \lambda\left(\lambda x_{j}\right)^{-(\alpha+1)}\left(1+\theta\left(\lambda x_{j}\right)^{-\alpha}\right)^{-(\beta+1)}\right]^{\delta_{a j}}\left[1-\left(1+\theta\left(\lambda x_{r a}\right)^{-\alpha}\right)^{-\beta}\right]^{1-\delta_{a j}}
\end{aligned}
$$

And the total likelihood function for $\left(t_{1}, \delta_{u 1}, \ldots, t_{n(1-\pi)}\right.$, $\left.\delta_{u n(1-\pi)}, x_{1}, \delta_{a 1}, \ldots, x_{n \pi}, \delta_{a n \pi}\right)$ is as follows:

$$
\begin{aligned}
& L(t, x \mid \alpha, \beta, \theta, \lambda)=L_{u i}\left(t_{i}, \delta_{u i} \mid \alpha, \beta, \theta\right) \cdot L_{a j}\left(x_{j}, \delta_{a j} \mid \alpha, \beta, \theta, \lambda\right) \\
& =\prod_{i=1}^{n(1-\pi)}\left[\alpha \beta \theta t_{i}^{-(\alpha+1)}\left(1+\theta t_{i}^{-\alpha}\right)^{-(\beta+1)}\right]^{\delta_{u i}}\left[1-\left(1+\theta t_{r u}^{-\alpha}\right)^{-\beta}\right]^{1-\delta_{u i}} \\
& * \prod_{j=1}^{n \pi}\left[\alpha \beta \theta \lambda\left(\lambda x_{j}\right)^{-(\alpha+1)}\left(1+\theta\left(\lambda x_{j}\right)^{-\alpha}\right)^{-(\beta+1)}\right]^{\delta_{u j}}\left[1-\left(1+\theta\left(\lambda x_{r a}\right)^{-\alpha}\right)^{-\beta}\right]^{1-\delta_{a j}}
\end{aligned}
$$

Where:

$$
\begin{aligned}
\delta_{u i} & =\left\{\begin{array}{lc}
1 & t_{i} \leq t_{r u} \\
0 & \text { otherwise }
\end{array} \quad i=1,2, \ldots, n(1-\pi)\right. \\
\delta_{a j} & =\left\{\begin{array}{cc}
1 & x_{j} \leq x_{r a} \\
0 & \text { otherwise }
\end{array} \quad j=1,2, \ldots, n \pi\right.
\end{aligned}
$$

It is usually easier to maximize the natural logarithm of the likelihood function rather than the likelihood function itself. We can get the natural logarithm of the likelihood function as follows:

$$
\begin{aligned}
& \ln L(\underset{-}{t, x} \mid \alpha, \beta, \theta, \lambda)=\left(r_{u}+r_{a}\right)(\ln \alpha+\ln \beta+\ln \theta) \\
& -r_{a} \alpha \ln \lambda-\sum_{i=1}^{r_{u}}\left[(\alpha+1) \ln t_{i}+(\beta+1) \ln \left(1+\theta t_{i}^{-\alpha}\right)\right] \\
& +n_{s u} \ln \left(1-\left(1+\theta t_{r u}^{-\alpha}\right)^{-\beta}\right) \\
& -\sum_{j=1}^{r_{a}}\left[(\alpha+1) \ln x_{j}+(\beta+1) \ln \left(1+\theta\left(\lambda x_{j}\right)^{-\alpha}\right)\right] \\
& +n_{s a} \ln \left(1-\left(1+\theta\left(\lambda x_{r a}\right)^{-\alpha}\right)^{-\beta}\right)
\end{aligned}
$$

Where:

$$
\begin{aligned}
& n_{s u}=n(1-\pi)-r_{u} \\
& n_{s a}=n \pi-r_{a}
\end{aligned}
$$

The first derivatives of the natural logarithm of the total likelihood function in (15) with respect to $\alpha, \beta, \theta$ and $\lambda$ are given by:

$$
\begin{aligned}
& \frac{\partial \ln L}{\partial \alpha}=\frac{\left(r_{u}+r_{a}\right)}{\alpha}-r_{a} \ln \lambda-\sum_{i=1}^{r_{u}}\left[\ln t_{i}-\frac{(\beta+1) \theta t_{i}^{-\alpha} \ln t_{i}}{\left(1+\theta t_{i}^{-\alpha}\right)}\right] \\
& -\frac{n_{s u} \beta \theta\left(1+\theta t_{r u}^{-\alpha}\right)^{-(\beta+1)} t_{r u}^{-\alpha} \ln t_{r u}}{1-\left(1+\theta t_{r u}^{-\alpha}\right)^{-\beta}} \\
& -\sum_{j=1}^{r_{a}}\left[\ln x_{j}-\frac{(\beta+1) \theta\left(\lambda x_{j}\right)^{-\alpha} \ln \left(\lambda x_{j}\right)}{\left(1+\theta\left(\lambda x_{j}\right)^{-\alpha}\right)}\right] \\
& -\frac{n_{s a} \beta \theta\left(1+\theta\left(\lambda x_{r a}\right)^{-\alpha}\right)^{-(\beta+1)}\left(\lambda x_{r a}\right)^{-\alpha} \ln \left(\lambda x_{r a}\right)}{1-\left(1+\theta\left(\lambda x_{r a}\right)^{-\alpha}\right)^{-\beta}} \\
& \frac{\partial \ln L}{\partial \beta}=\frac{\left(r_{u}+r_{a}\right)}{\beta}-\sum_{i=1}^{r_{r u}}\left[\ln \left(1+\theta t_{i}^{-\alpha}\right)\right] \\
& +\frac{n_{s u}\left(1+\theta t_{r u}^{-\alpha}\right)^{-\beta} \ln \left(1+\theta t_{r u}^{-\alpha}\right)}{1-\left(1+\theta t_{r u}^{-\alpha}\right)^{-\beta}} \\
& -\sum_{j=1}^{r_{a}}\left[\ln \left(1+\theta\left(\lambda x_{j}\right)^{-\alpha}\right)\right]+\frac{n_{s a}\left(1+\theta\left(\lambda x_{r a}\right)^{-\alpha}\right)^{-\beta} \ln \left(1+\theta\left(\lambda x_{r a}\right)^{-\alpha}\right)}{1-\left(1+\theta\left(\lambda x_{r a}\right)^{-\alpha}\right)^{-\beta}}
\end{aligned}
$$




$$
\begin{aligned}
& \frac{\partial \ln L}{\partial \theta}=\frac{\left(r_{u}+r_{a}\right)}{\theta}-\sum_{i=1}^{r_{u}}\left[\frac{(\beta+1) t_{i}^{-\alpha}}{\left(1+\theta t_{i}^{-\alpha}\right)}\right]+\frac{n_{s u} \beta\left(1+\theta t_{r u}^{-\alpha}\right)^{-(\beta+1)} t_{r u}^{-\alpha}}{1-\left(1+\theta t_{r u}^{-\alpha}\right)^{-\beta}} \\
& -\sum_{j=1}^{r_{a}}\left[\frac{(\beta+1)\left(\lambda x_{j}\right)^{-\alpha}}{\left(1+\theta\left(\lambda x_{j}\right)^{-\alpha}\right)}\right]+\frac{n_{s a} \beta\left(1+\theta\left(\lambda x_{r a}\right)^{-\alpha}\right)^{-(\beta+1)}\left(\lambda x_{r a}\right)^{-\alpha}}{1-\left(1+\theta\left(\lambda x_{r a}\right)^{-\alpha}\right)^{-\beta}} \\
& \frac{\partial \ln L}{\partial \lambda}=\frac{-r_{a} \alpha}{\lambda}+\sum_{j=1}^{r_{a}}\left[\frac{\alpha \theta(\beta+1)\left(x_{j}\right)^{-\alpha} \lambda^{-(\alpha+1)}}{\left(1+\theta\left(\lambda x_{j}\right)^{-\alpha}\right)}\right] \\
& -\frac{n_{s a} \beta \alpha \theta\left(1+\theta\left(\lambda x_{r a}\right)^{-\alpha}\right)^{-(\beta+1)}\left(x_{r a}\right)^{-\alpha} \lambda^{-(\alpha+1)}}{1-\left(1+\theta\left(\lambda x_{r a}\right)^{-\alpha}\right)^{-\beta}}
\end{aligned}
$$

Therefore, the maximum likelihood estimates of $\alpha$, $\beta, \theta$ and $\lambda$ are obtained by setting Equations (16), (17), (18) and (19) to be equal zero. Obviously, it is very difficult to obtain a closed form solution for the four non-linear equations. So, iterative procedures will be used to solve these equations numerically. NewtonRaphson method is used to get the maximum likelihood estimators of $\alpha, \beta, \theta$ and $\lambda$.

Concerning the asymptotic variance-covariance matrix of the ML estimators of the parameters, it can be approximated by numerically inverting the Fisherinformation matrix F. It is composed of the negative second derivatives of the natural logarithm of the likelihood function evaluated at the ML estimates. Therefore, the asymptotic Fisher-information matrix can be written as follows:

$$
\begin{aligned}
& \frac{\partial^{2} \ln L}{\partial \alpha \partial \beta}=\sum_{i=1}^{r_{u}}\left[\frac{\theta t_{i}^{-\alpha}\left(\ln t_{i}\right)}{\left(1+\theta t_{i}^{-\alpha}\right)}\right]+\sum_{j=1}^{r_{a}}\left[\frac{\theta\left(\lambda x_{j}\right)^{-\alpha} \ln \left(\lambda x_{j}\right)}{\left(1+\theta\left(\lambda x_{j}\right)^{-\alpha}\right)}\right] \\
& =\frac{n_{s u} \theta t_{r u}^{-\alpha}\left(\ln t_{r u}\right)\left(1+\theta t_{r u}^{-\alpha}\right)^{-(\beta+1)}\left(1-\beta \ln \left(1+\theta t_{r u}^{-\alpha}\right)-\left(1+\theta t_{r u}^{-\alpha}\right)^{-\beta}\right)}{\left(1-\left(1+\theta t_{r u}^{-\alpha}\right)^{-\beta}\right)^{2}} \\
& -\frac{n_{s a} \theta\left(\lambda x_{r a}\right)^{-\alpha} \ln \left(\lambda x_{r a}\right)\left(1+\theta\left(\lambda x_{r a}\right)^{-\alpha}\right)^{-(\beta+1)}\left(1-\beta \ln \left(1+\theta\left(\lambda x_{r a}\right)^{-\alpha}\right)-\left(1+\theta\left(\lambda x_{r a}\right)^{-\alpha}\right)^{-\beta}\right)}{\left(1-\left(1+\theta\left(\lambda x_{r a}\right)^{-\alpha}\right)^{-\beta}\right)^{2}}
\end{aligned}
$$

where

$\Psi_{u}=\left(1+\theta t_{r u}^{-\alpha}\right)^{-\beta}+\beta \theta t_{r u}^{-\alpha}-1$

$\Psi_{a}=\left(1+\theta\left(\lambda x_{r a}\right)^{-\alpha}\right)^{-\beta}+\beta \theta\left(\lambda x_{r a}\right)^{-\alpha}-1$

$$
\begin{aligned}
& \frac{\partial^{2} \ln L}{\partial \alpha \partial \theta}=\sum_{i=1}^{r_{u}}\left[\frac{(\beta+1) t_{i}^{-\alpha}\left(\ln t_{i}\right)}{\left(1+\theta t_{i}^{-\alpha}\right)^{2}}\right]+\sum_{j=1}^{r_{a}}\left[\frac{(\beta+1)\left(\lambda x_{j}\right)^{-\alpha} \ln \left(\lambda x_{j}\right)}{\left(1+\theta\left(\lambda x_{j}\right)^{-\alpha}\right)^{2}}\right] \\
& -\frac{n_{s u} \beta t_{r u}^{-\alpha}\left(\ln t_{r u}\right)\left(1+\theta t_{r u}^{-\alpha}\right)^{-(\beta+2)}\left(1-\theta \beta t_{r u}^{-\alpha}-\left(1+\theta t_{r u}^{-\alpha}\right)^{-\beta}\right)}{\left(1-\left(1+\theta t_{r u}^{-\alpha}\right)^{-\beta}\right)^{2}} \\
& -\frac{n_{s a} \beta\left(\lambda x_{r a}\right)^{-\alpha} \ln \left(\lambda x_{r a}\right)\left(1+\theta\left(\lambda x_{r a}\right)^{-\alpha}\right)^{-(\beta+2)}\left(1-\beta \theta\left(\lambda x_{r a}\right)^{-\alpha}-\left(1+\theta\left(\lambda x_{r a}\right)^{-\alpha}\right)^{-\beta}\right)}{\left(1-\left(1+\theta\left(\lambda x_{r a}\right)^{-\alpha}\right)^{-\beta}\right)^{2}}
\end{aligned}
$$


Eman A. El-Dessouky Nassef / Journal of Mathematics and Statistics 2020, Volume 16: 148.160 DOI: $10.3844 / j m s s p .2020 .148 .160$

$$
\begin{aligned}
& \frac{\partial^{2} \ln L}{\partial \alpha \partial \lambda}=\frac{-r_{a}}{\lambda}+\sum_{j=1}^{r a}\left[\frac{(\beta+1) \theta\left(x_{j}\right)^{-\alpha} \lambda^{-(\alpha+1)}\left[1-\alpha \ln \left(\lambda x_{j}\right)+\theta\left(\lambda x_{j}\right)^{-\alpha}\right]}{\left(1+\theta\left(\lambda x_{j}\right)^{-\alpha}\right)^{2}}\right] \\
& -\frac{n_{s a} \beta \theta\left(x_{r a}\right)^{-\alpha} \lambda^{-(\alpha+1)}\left(1+\theta\left(\lambda x_{r a}\right)^{-\alpha}\right)^{-(\beta+2)} \phi_{a}}{\left(1-\left(1+\theta\left(\lambda x_{r a}\right)^{-\alpha}\right)^{-\beta}\right)^{2}} \\
& \phi_{a}=\left(1+\theta\left(\lambda x_{r a}\right)^{-\alpha}\right)\left(1-\alpha \ln \left(\lambda x_{r a}\right)\right)+\alpha \theta(\beta+1)\left(\lambda x_{r a}\right)^{-\alpha} \ln \left(\lambda x_{r a}\right) \\
& +\left(1+\theta\left(\lambda x_{r a}\right)^{-\alpha}\right)^{-\beta+1}\left(\alpha \ln \left(\lambda x_{r a}\right)-1\right)-\alpha \theta\left(1+\theta\left(\lambda x_{r a}\right)^{-\alpha}\right)^{-\beta}\left(\lambda x_{r a}\right)^{-\alpha} \ln \left(\lambda x_{r a}\right) \\
& \frac{\partial^{2} \ln L}{\partial \beta^{2}}=\frac{-\left(r_{u}+r_{a}\right)}{\beta^{2}}-\frac{n_{s u}\left(\ln \left(1+\theta t_{r u}^{-\alpha}\right)\right)^{2}\left(1+\theta t_{r u}^{-\alpha}\right)^{-\beta}}{\left(1-\left(1+t_{r u}^{-\alpha}\right)^{-\beta}\right)^{2}} \\
& -\frac{n_{s a}\left(\ln \left(1+\theta\left(\lambda x_{r a}\right)^{-\alpha}\right)\right)^{2}\left(1+\theta\left(\lambda x_{r a}\right)^{-\alpha}\right)^{-\beta}}{\left(1-\left(1+\theta\left(\lambda x_{r a}\right)^{-\alpha}\right)^{-\beta}\right)^{2}} \\
& \frac{\partial^{2} \ln L}{\partial \beta \partial \theta}=-\sum_{i=1}^{r_{u}}\left[\frac{t_{i}^{-\alpha}}{\left(1+\theta t_{i}^{-\alpha}\right)}\right]-\sum_{j=1}^{r_{a}}\left[\frac{\left(\lambda x_{j}\right)^{-\alpha}}{\left(1+\theta\left(\lambda x_{j}\right)^{-\alpha}\right)}\right] \\
& +\frac{n_{s u} t_{r u}^{-\alpha}\left(1+\theta t_{r u}^{-\alpha}\right)^{-(\beta+1)}\left(1-\beta \ln \left(1+\theta t_{r u}^{-\alpha}\right)-\left(1+\theta t_{r u}^{-\alpha}\right)^{-\beta}\right)}{\left(1-\left(1+\theta t_{r u}^{-\alpha}\right)^{-\beta}\right)^{2}} \\
& +\frac{n_{s a}\left(\lambda x_{r a}\right)^{-\alpha}\left(1+\theta\left(\lambda x_{r a}\right)^{-\alpha}\right)^{-(\beta+1)}\left(1-\beta \ln \left(1+\theta\left(\lambda x_{r a}\right)^{-\alpha}\right)-\left(1+\theta\left(\lambda x_{r a}\right)^{-\alpha}\right)^{-\beta}\right)}{\left(1-\left(1+\theta\left(\lambda x_{r a}\right)^{-\alpha}\right)^{-\beta}\right)^{2}} \\
& \frac{\partial^{2} \ln L}{\partial \beta \partial \lambda}=\sum_{j=1}^{r_{a}}\left[\frac{\alpha \theta\left(x_{j}\right)^{-\alpha} \lambda^{-(\alpha+1)}}{\left(1+\theta\left(\lambda x_{j}\right)^{-\alpha}\right)}\right]+\frac{n_{s a} \alpha \theta\left(x_{r a}\right)^{-\alpha} \lambda^{-(\alpha+1)}\left(1+\theta\left(\lambda x_{r a}\right)^{-\alpha}\right)^{-(\beta+1)} \varpi_{a}}{\left(1-\left(1+\theta\left(\lambda x_{r a}\right)^{-\alpha}\right)^{-\beta}\right)^{2}} \\
& \varpi_{a}=\beta \ln \left(1+\theta\left(\lambda x_{r a}\right)^{-\alpha}\right)+\left(1+\theta\left(\lambda x_{r a}\right)^{-\alpha}\right)^{-\beta}-1 \\
& \frac{\partial^{2} \ln L}{\partial \theta^{2}}=\sum_{i=1}^{r_{u}}\left[\frac{(\beta+1) t_{i}^{-2 \alpha}}{\left(1+\theta t_{i}^{-\alpha}\right)^{2}}\right]+\sum_{j=1}^{r_{a}}\left[\frac{(\beta+1)\left(\lambda x_{j}\right)^{-2 \alpha}}{\left(1+\theta\left(\lambda x_{j}\right)^{-\alpha}\right)^{2}}\right]-\frac{\left(r_{u}+r_{a}\right)}{\theta^{2}} \\
& +\frac{n_{s u} \beta t_{r u}^{-2 \alpha}\left(1+\theta t_{r u}^{-\alpha}\right)^{-(\beta+2)}\left(\left(1+\theta t_{r u}^{-\alpha}\right)^{-\beta}-(\beta+1)\right)}{\left(1-\left(1+\theta t_{r u}^{-\alpha}\right)^{-\beta}\right)^{2}} \\
& +\frac{n_{s a} \beta\left(\lambda x_{r a}\right)^{-2 \alpha}\left(1+\theta\left(\lambda x_{r a}\right)^{-\alpha}\right)^{-(\beta+2)}\left(\left(1+\theta\left(\lambda x_{r a}\right)^{-\alpha}\right)^{-\beta}-(\beta+1)\right)}{\left(1-\left(1+\theta\left(\lambda x_{r a}\right)^{-\alpha}\right)^{-\beta}\right)^{2}}
\end{aligned}
$$

153 


$$
\begin{aligned}
& \frac{\partial^{2} \ln L}{\partial \theta \partial \lambda}=\sum_{j=1}^{r_{a}}\left[\frac{\alpha(\beta+1)\left(x_{j}\right)^{-\alpha} \lambda^{-(\alpha+1)}}{\left(1+\theta\left(\lambda x_{j}\right)^{-\alpha}\right)^{2}}\right] \\
& -\frac{n_{s a} \alpha \beta\left(x_{r a}\right)^{-\alpha} \lambda^{-(\alpha+1)}\left(1+\theta\left(\lambda x_{r a}\right)^{-\alpha}\right)^{-(\beta+2)} \rho_{a}}{\left(1-\left(1+\theta\left(\lambda x_{r a}\right)^{-\alpha}\right)^{-\beta}\right)^{2}} \\
& \rho_{a}=\left(1+\theta\left(\lambda x_{r a}\right)^{-\alpha}\right)-\theta(\beta+1)\left(\lambda x_{r a}\right)^{-\alpha}-\left(1+\theta\left(\lambda x_{r a}\right)^{-\alpha}\right)^{-\beta+1} \\
& +\theta\left(\lambda x_{r a}\right)^{-\alpha}\left(1+\theta\left(\lambda x_{r a}\right)^{-\alpha}\right)^{-\beta} \\
& \frac{\partial^{2} \ln L}{\partial \lambda^{2}}=\frac{\alpha r_{a}}{\lambda^{2}}-\sum_{j=1}^{r_{a}}\left[\frac{\alpha \theta(\beta+1)\left(x_{j}\right)^{-\alpha} \lambda^{-(\alpha+2)}\left[(\alpha+1)+\theta\left(\lambda x_{j}\right)^{-\alpha}\right]}{\left(1+\theta\left(\lambda x_{j}\right)^{-\alpha}\right)^{2}}\right] \\
& -\frac{n_{s a} \alpha \beta \theta\left(x_{r a}\right)^{-\alpha} \lambda^{-(\alpha+2)}\left(1+\theta\left(\lambda x_{r a}\right)^{-\alpha}\right)^{-(\beta+2)} \zeta_{a}}{\left(1-\left(1+\theta\left(\lambda x_{r a}\right)^{-\alpha}\right)^{-\beta}\right)^{2}} \\
& \zeta_{a}=\alpha \theta(\beta+1)\left(\lambda x_{r a}\right)^{-\alpha}-(\alpha+1)\left(1+\theta\left(\lambda x_{r a}\right)^{-\alpha}\right)+(\alpha+1)\left(1+\theta\left(\lambda x_{r a}\right)^{-\alpha}\right)^{-\beta+1} \\
& -\theta \alpha\left(\lambda x_{r a}\right)^{-\alpha}\left(1+\left(\lambda x_{r a}\right)^{-\alpha}\right)^{-\beta}
\end{aligned}
$$

So, an asymptotic variance covariance matrix $V=F^{-1}$ defined by inverting the Fisher information matrix $F$ and substituting $\hat{\alpha}$ for $\alpha, \hat{\beta}$ for $\beta, \hat{\theta}$ for $\theta$ and $\hat{\lambda}$ for $\lambda$.

\section{Confidence Interval for the Model Parameters}

We can get the Confidence Interval for the model parameters by using two methods, first by depending on normal approximation and the second by using bootstrap method.

\section{Normal Approximation Method}

The maximum likelihood estimates $\hat{\alpha}, \hat{\beta}, \hat{\theta}$ and $\hat{\lambda}$ in large samples, are consistent and asymptotically normally distributed. Therefore, the two sided approximate $100 \gamma \%$ confidence limits for the maximum likelihood estimates $\hat{\alpha}, \hat{\beta}, \hat{\theta}$ and $\hat{\lambda}$ can be obtained, respectively, as follows:

$$
\begin{array}{ll}
L_{\hat{\alpha}}=\hat{\alpha}-z_{\gamma / 2} \sigma(\hat{\alpha}) & , \mathrm{U}_{\hat{\alpha}}=\hat{\alpha}+z_{\gamma / 2} \sigma(\hat{\alpha}) \\
L_{\hat{\beta}}=\hat{\beta}-z_{\gamma / 2} \sigma(\hat{\beta}) & , \mathrm{U}_{\hat{\beta}}=\hat{\beta}+z_{\gamma / 2} \sigma(\hat{\beta}) \\
L_{\hat{\theta}}=\hat{\theta}-z_{\gamma / 2} \sigma(\hat{\theta}) & , \mathrm{U}_{\hat{\theta}}=\hat{\theta}+z_{\gamma / 2} \sigma(\hat{\theta})
\end{array}
$$

and

$L_{\hat{\lambda}}=\hat{\lambda}-z_{\gamma / 2} \sigma(\hat{\lambda}) \quad, \mathrm{U}_{\hat{\lambda}}=\hat{\lambda}+z_{\gamma / 2} \sigma(\hat{\lambda})$

where $\quad z_{\gamma / 2}$ is the $\left[\frac{100(1-\gamma)}{2}\right]^{\text {th }}$ standard normal percentile and $\sigma($.$) is the standard deviation for the$ maximum likelihood estimates which is obtained by taking square root of the first diagonal element of $F^{-1}$.

therefore, the two sided approximate confidence limits for $\alpha, \beta, \theta$ and $\lambda$ will be constructed with confidence levels $95 \%$.

\section{Bootstrap Method}

Bootstrapping is any test that relies on random sampling with replacement. Bootstrapping allows assigning measures of accuracy to sample estimates. This technique allows estimation of the sampling distribution of almost any statistic using random sampling methods (Al-Zahrani, 2016; Young et al., 2007).

Confidence intervals carried out based on the asymptotic results are usually expected not to perform well. Therefore, we propose to use confidence intervals based on the percentile bootstrap method.

The algorithms for estimating the confidence intervals of $\alpha, \beta, \theta$ and $\lambda$ using the percentile bootstrap method as follow:

1. Use the samples $t_{(1)}<t_{(2)}<\cdots<t_{(r u)}, x_{(1)}<x_{(2)}<\cdots<$ $x_{(r a)}$ and Equations (16), (17), (18) and (19) to compute the estimates of maximum likelihood $\hat{\alpha}, \hat{\beta}, \hat{\theta}$ and $\hat{\lambda}$

2. Use the estimates $\hat{\alpha}, \hat{\beta}, \hat{\theta}$ and $\hat{\lambda}$ to generate a bootstrap sample $t_{(1)}<t_{(2)}<\cdots<t_{(r u)}, x_{(1)}<x_{(2)}<\cdots<x_{(r a)}$

3. Repeat Step 2, 1000 BOOT times

4. we can get four vectors of MLs for each parameter which contains 1000 observations 
Then the approximate $100(1-\gamma) \%$ confidence intervals for $\alpha, \beta, \theta$ and $\lambda$ are given by arranging the values in ascending order to obtain the confidence intervals as:

$$
\begin{aligned}
& {\left[\hat{\alpha}^{*}(\gamma), \hat{\alpha}^{*}(1-\gamma)\right],\left[\hat{\beta}^{*}(\gamma), \hat{\beta}^{*}(1-\gamma)\right],} \\
& {\left[\hat{\theta}^{*}(\gamma), \hat{\theta}^{*}(1-\gamma)\right] \text { and }\left[\hat{\lambda}^{*}(\gamma), \hat{\lambda}^{*}(1-\gamma)\right] .}
\end{aligned}
$$

\section{Numerical Study}

A simulation study is carried out to investigate the performance of the estimators for items having Dagum distribution based on type II censored samples. The performance of estimators has been considered in terms of their Relative Absolute Bias (RAB) and Means Square Error (MSE).

A simulation study is performed according to the following steps:

Step 1: Divided the total sample size $n$ into two subsamples $n_{a}$ and $n_{u}$ sample size of accelerated condition items $n_{a}=n \pi$ and sample size of normal condition items $n_{u}=n(1-\pi)$

Where $\pi$ proportion of accelerated condition is equal 0.5 .

Step 2: Our experiment is done under type II censoring which means the experiment terminate when reaching the first number failures $r_{u}$ and $r_{a}$ for normal and accelerated conditions respectively.

Generate random samples of size $r_{u}=0.8 n_{u}$ as normal condition samples from Dagum distribution. Furthermore, Generate random samples of size $r_{a}=0.8 n_{a}$ as stress condition samples from Dagum distribution.

Step 3: 1000 random samples of sizes 200, 300 and 400 are generated from Dagum distribution. The parameters' values are chosen as:

Case 1: $\alpha=2, \beta=1.8, \theta=1.6$ and $\lambda=1.4$

Case 2: $\alpha=2, \beta=1.8, \theta=1.6$ and $\lambda=2$

Case 3: $\alpha=2, \beta=1.8, \theta=1.9$ and $\lambda=1.4$

Case 4: $\alpha=2, \beta=2.2, \theta=1.9$ and $\lambda=1.4$

Case 5: $\alpha=2.5, \beta=2, \theta=1.9$ and $\lambda=1.4$

Case 6: $\alpha=2.5, \beta=2.2, \theta=1.9$ and $\lambda=2$

Step 4: For each sample and for the selected sets of parameters, the distribution parameters $\alpha, \beta$ and $\theta$ and the acceleration factor $\lambda$ are estimated in CSPALT under type II censored samples. Newton -Raphson technique is applied for solving the nonlinear Equations (16), (17), (18) and (19) to get the estimates of $\alpha, \beta, \theta$ and $\lambda$.
Step 5: The Relative Bias and MSE of the estimators for the distribution parameters and acceleration factor for all sample sizes are computed.

Step 6: The asymptotic variance and covariance matrix of the estimators for different sample sizes are obtained by using Equations (21-30).

Step 7: Using normal approximation method to construct the two sided confidence limits with confidence levels and of the acceleration factor and the two parameters are constructed using Equation (31) for $\gamma=95 \%$.

Step8: Using Boot strap method to construct the two sided confidence limits for parameters $\alpha, \beta, \theta$ and $\lambda$ at $\gamma=95 \%$.

Step 9: Using the estimated parameters and confidence limits to predict warrant cost models as in Equations (5), (6) and 7 for FRW and (8), (9), (10) for PRW at $t_{w f}=3, t_{w p}=6, C_{w}=100$.

Our simulation results are summarized in Table 1 to 4 .

Table 1 and 2 give the Maximum Likelihood Estimators (MLE) for Dagum distribution parameters and The accelerated factor, Means Square Error (MSE), Relative Absolute Bias (RAB) and asymptotic variance and covariance matrix of estimators are calculated for the selected set of parameters and different sample sizes.

In Table 3, Confidence interval for model parameters are constructed at confidence level $95 \%$ using normal approximation and percentile Bootstrap methods. Table 4 shows the expected and confidence interval for warranty cost models at confidence level $95 \%$ for two types of warranty models FRW and PRW.

From these tables, the following observations can be made on the performance of estimated parameters of Dagum distribution based on partially accelerated life testing with constant stress and type II censored samples:

1. It is observed that The MSE and RAB of $\alpha$ decreases as the increasing the values of $\beta, \theta$ and $\lambda$ but increases with the increasing values of $\alpha$. There are direct relationship between MSE and $\mathrm{RAB}$ of $\beta$ and the values of $\beta$ and $\lambda$ while inverse relationship between the values of $\alpha$ and $\theta$. The MSE of $\theta$ increases as increases the values of $\beta, \theta$ and $\lambda$ where decreases as increasing the value of $\alpha$ and inverse relation between RAB of $\theta$ and the values of $\alpha, \beta$ and $\lambda$. finally, there are inverse relation between the MSE and RAB of $\lambda$ and the values of $\alpha, \beta$ and $\theta$

2. The values of the MSE and RAB of the estimates of $\alpha, \beta, \theta$ and $\lambda$ decreases as the sample size 
increases. The estimates obtained by the present model estimates the true parameters $\alpha, \beta, \theta$ and $\lambda$ quite well respectively with relatively small mean squared errors

3. The asymptotic variances of the estimates as in Table 2 decrease as the sample size increases, therefore the confidence interval length for the estimated parameters (Table 3) whether in normal approximation or Bootstrap method decreases. The expected warranty cost and its confidence intervals (Table 4) increases for two types of warranty FRW and PRW
4. In general, there are convergence between two methods of confidence interval for estimated parameters. By Studying the effect of parameters on the length of confidence intervals, we find there are inverse relationship between the values of $\alpha$ and $\beta$ and the length of confidence interval $\lambda$, also the increasing the value of $\alpha$ is not effect on the length of confidence interval of $\beta$ and $\theta$

5. The expected warranty cost and its confidence intervals for two types of warranty FRW and PRW are in direct relationship with the values of $\alpha$ while the inverse relation with the values of $\beta$ and $\theta$

Table 1: The MLE, relative absolute bias and MSE of the estimators

\begin{tabular}{|c|c|c|c|c|c|c|c|}
\hline \multirow[b]{2}{*}{$\mathrm{n}$} & \multirow[b]{2}{*}{ parameter } & \multicolumn{3}{|c|}{$\alpha=2, \beta=1.8, \theta=1.6$ and $\lambda=1.4$} & \multicolumn{3}{|c|}{$\alpha=2, \beta=1.8, \theta=1.6$ and $\lambda=1.4$} \\
\hline & & MLE & MSE & RAB & MLE & MSE & RAB \\
\hline \multirow[t]{4}{*}{200} & $\alpha$ & 2.020049 & 0.02007 & 0.010024 & 2.019633 & 0.020056 & 0.009817 \\
\hline & $\beta$ & 1.866981 & 0.15004 & 0.037211 & 1.869039 & 0.151214 & 0.038355 \\
\hline & $\theta$ & 1.659181 & 0.254676 & 0.036988 & 1.657484 & 0.256648 & 0.035928 \\
\hline & $\lambda$ & 1.41077 & 0.022759 & 0.007692 & 2.015331 & 0.046467 & 0.007665 \\
\hline \multirow[t]{4}{*}{300} & $\alpha$ & 2.01687 & 0.013977 & 0.008435 & 2.01689 & 0.014016 & 0.008445 \\
\hline & $\beta$ & 1.841795 & 0.088768 & 0.023219 & 1.841867 & 0.088956 & 0.023259 \\
\hline & $\theta$ & 1.644075 & 0.165748 & 0.027547 & 1.644255 & 0.166383 & 0.027659 \\
\hline & $\lambda$ & 1.404069 & 0.013595 & 0.002907 & 2.005801 & 0.027747 & 0.0272 \\
\hline \multirow[t]{5}{*}{400} & $\alpha$ & 2.012554 & 0.010643 & 0.006277 & 2.012333 & 0.010662 & 0.006167 \\
\hline & $\beta$ & 1.833054 & 0.067336 & 0.018363 & 1.83403 & 0.067653 & 0.018906 \\
\hline & $\theta$ & 1.640847 & 0.118387 & 0.025529 & 1.639775 & 0.118944 & 0.024859 \\
\hline & $\lambda$ & 1.410812 & 0.010099 & 0.007723 & 2.015413 & 0.020607 & 0.007706 \\
\hline & & $\alpha=2, \beta=$ & .9 and $\lambda=2$ & & $\alpha=2, \beta=$ & $=1.9$ and $\lambda$ & \\
\hline \multirow[t]{4}{*}{200} & $\alpha$ & 2.019331 & 0.019038 & 0.009665 & 2.018066 & 0.017192 & 0.009033 \\
\hline & $\beta$ & 1.864972 & 0.141751 & 0.036095 & 2.297833 & 0.250202 & 0.044469 \\
\hline & $\theta$ & 1.971179 & 0.353924 & 0.037463 & 1.9677 & 0.373543 & 0.035632 \\
\hline & $\lambda$ & 2.015429 & 0.046489 & 0.007715 & 2.014611 & 0.042127 & 0.007305 \\
\hline \multirow[t]{4}{*}{300} & $\alpha$ & 2.016321 & 0.013272 & 0.008161 & 2.01542 & 0.012082 & 0.00771 \\
\hline & $\beta$ & 1.841021 & 0.084715 & 0.022789 & 2.260236 & 0.144064 & 0.02738 \\
\hline & $\theta$ & 1.952692 & 0.229935 & 0.027732 & 1.950601 & 0.24024 & 0.026632 \\
\hline & $\lambda$ & 2.005801 & 0.027745 & 0.002901 & 2.005059 & 0.025364 & 0.002529 \\
\hline \multirow[t]{5}{*}{400} & $\alpha$ & 2.011884 & 0.010185 & 0.005942 & 2.011101 & 0.009344 & 0.005551 \\
\hline & $\beta$ & 1.83364 & 0.064828 & 0.018689 & 2.250127 & 0.11269 & 0.022785 \\
\hline & $\theta$ & 1.947409 & 0.165429 & 0.024952 & 1.944821 & 0.17414 & 0.02359 \\
\hline & $\lambda$ & 2.015422 & 0.020622 & 0.007711 & 2.014518 & 0.018855 & 0.007259 \\
\hline & & $\alpha=2.5, \beta$ & .9 and $\lambda=2$ & & $\alpha=2.5, \beta$ & $\theta=1.9$ and & \\
\hline \multirow[t]{4}{*}{200} & $\alpha$ & 2.523477 & 0.028022 & 0.00939 & 2.522415 & 0.026864 & 0.008966 \\
\hline & $\beta$ & 2.077696 & 0.1847852 & 0.038848 & 2.298209 & 0.249899 & 0.044641 \\
\hline & $\theta$ & 1.969892 & 0.357062 & 0.036785 & 1.966643 & 0.372131 & 0.035075 \\
\hline & $\lambda$ & 2.010202 & 0.028000 & 0.0051012 & 2.010013 & 0.026768 & 0.005067 \\
\hline \multirow[t]{4}{*}{300} & $\alpha$ & 2.520021 & 0.019756 & 0.008008 & 2.519142 & 0.01872 & 0.007657 \\
\hline & $\beta$ & 2.04933 & 0.11159 & 0.024665 & 2.260149 & 0.142877 & 0.02734 \\
\hline & $\theta$ & 1.952865 & 0.234911 & 0.027823 & 1.949763 & 0.23849 & 0.026191 \\
\hline & $\lambda$ & 2.003269 & 0.016896 & 0.001634 & 2.003012 & 0.016208 & 0.001506 \\
\hline \multirow[t]{4}{*}{400} & $\alpha$ & 2.514608 & 0.015148 & 0.005843 & 2.513904 & 0.014537 & 0.005562 \\
\hline & $\beta$ & 2.039924 & 0.085105 & 0.019961 & 2.249964 & 0.112395 & 0.022711 \\
\hline & $\theta$ & 1.947312 & 0.167476 & 0.024901 & 1.944765 & 0.173582 & 0.023561 \\
\hline & $\lambda$ & 2.011242 & 0.012506 & 0.005620 & 2.010867 & 0.011999 & 0.005433 \\
\hline
\end{tabular}


Eman A. El-Dessouky Nassef / Journal of Mathematics and Statistics 2020, Volume 16: 148.160 DOI: 10.3844/jmssp.2020.148.160

Table 2: Asymptotic variance and covariance matrix of estimators

\begin{tabular}{|c|c|c|c|c|c|c|c|c|c|}
\hline \multirow[b]{2}{*}{$\underline{\mathrm{n}}$} & \multirow[b]{2}{*}{ Parameter } & \multicolumn{4}{|c|}{$\alpha=2, \beta=1.8, \theta=1.6$ and $\lambda=1.4$} & \multicolumn{4}{|c|}{$\alpha=2, \beta=1.8, \theta=1.6$ and $\lambda=2$} \\
\hline & & $\hat{\alpha}$ & $\hat{\beta}$ & $\hat{\theta}$ & $\hat{\lambda}$ & $\hat{\alpha}$ & $\hat{\beta}$ & $\hat{\theta}$ & $\hat{\lambda}$ \\
\hline \multirow[t]{4}{*}{200} & $\hat{\alpha}$ & 0.015 & -0.0036 & 0.002 & -0.005 & 0.0148 & -0.004 & 0.0024 & -0.007 \\
\hline & $\hat{\beta}$ & -0.004 & 0.0274 & -0.007 & 0.0133 & -0.004 & 0.027 & -0.0069 & 0.019 \\
\hline & $\hat{\theta}$ & 0.002 & -0.0069 & 0.012 & 0.0004 & 0.0024 & -0.007 & 0.0122 & 0.0006 \\
\hline & $\hat{\lambda}$ & -0.005 & 0.0133 & 4E-04 & 0.0181 & -0.007 & 0.019 & 0.0006 & 0.0369 \\
\hline \multirow[t]{4}{*}{300} & $\hat{\alpha}$ & 0.01 & -0.0018 & 0.003 & -0.004 & 0.0099 & -0.002 & 0.0025 & -0.005 \\
\hline & $\hat{\beta}$ & -0.002 & 0.0139 & -0.005 & 0.0073 & -0.002 & 0.014 & -0.0053 & 0.0105 \\
\hline & $\hat{\theta}$ & 0.003 & -0.0053 & 0.016 & 0.0002 & 0.0025 & -0.005 & 0.0165 & 0.0003 \\
\hline & $\hat{\lambda}$ & -0.004 & 0.0073 & 2E-04 & 0.0111 & -0.005 & 0.01 & 0.0003 & 0.0226 \\
\hline \multirow[t]{5}{*}{400} & $\hat{\alpha}$ & 0.007 & -0.0012 & 0.001 & -0.002 & 0.0067 & -0.001 & 0.0013 & -0.003 \\
\hline & $\hat{\beta}$ & -0.001 & 0.0133 & -0.004 & 0.0068 & -0.001 & 0.013 & -0.0038 & 0.0097 \\
\hline & $\hat{\theta}$ & 0.001 & -0.0038 & 0.008 & 0.0004 & 0.0013 & -0.004 & 0.0079 & 0.0005 \\
\hline & $\hat{\lambda}$ & -0.002 & 0.0068 & $4 \mathrm{E}-04$ & 0.0104 & -0.003 & 0.01 & 0.0005 & 0.0212 \\
\hline & & \multicolumn{4}{|c|}{$\alpha=2, \beta=1.8, \theta=1.9$ and $\lambda=2$} & \multicolumn{4}{|c|}{$\alpha=2, \beta=2.2, \theta=1.9$ and $\lambda=2$} \\
\hline \multirow[t]{4}{*}{200} & $\hat{\alpha}$ & 0.014 & -0.0024 & 0.003 & -0.007 & 0.0129 & $-3 \mathrm{E}-04$ & 0.0028 & -0.005 \\
\hline & $\hat{\beta}$ & -0.002 & 0.0272 & -0.008 & 0.0186 & $-3 \mathrm{E}-04$ & 0.044 & -0.0116 & 0.0214 \\
\hline & $\hat{\theta}$ & 0.003 & -0.0081 & 0.017 & 0.0006 & 0.0028 & -0.012 & 0.0193 & 0.0006 \\
\hline & $\hat{\lambda}$ & -0.007 & 0.0186 & $6 \mathrm{E}-04$ & 0.037 & -0.005 & 0.021 & 0.0006 & 0.0331 \\
\hline \multirow[t]{4}{*}{300} & $\hat{\alpha}$ & 0.009 & -0.0012 & 0.003 & -0.005 & 0.0077 & $-1 \mathrm{E}-04$ & 0.002 & -0.004 \\
\hline & $\hat{\beta}$ & -0.001 & 0.014 & -0.006 & 0.0103 & $-1 \mathrm{E}-04$ & 0.037 & -0.0091 & 0.0162 \\
\hline & $\hat{\theta}$ & 0.003 & -0.0062 & 0.023 & 0.0003 & 0.002 & -0.009 & 0.0135 & $-3 E-06$ \\
\hline & $\hat{\lambda}$ & -0.005 & 0.0103 & $3 \mathrm{E}-04$ & 0.0226 & -0.004 & 0.016 & $-3 \mathrm{E}-06$ & 0.0219 \\
\hline \multirow[t]{5}{*}{400} & $\hat{\alpha}$ & 0.006 & -0.0007 & 0.002 & -0.003 & 0.0059 & $2 \mathrm{E}-04$ & 0.0016 & -0.003 \\
\hline & $\hat{\beta}$ & $-7 E-04$ & 0.0132 & -0.004 & 0.0095 & 0.0002 & 0.02 & -0.0061 & 0.0106 \\
\hline & $\hat{\theta}$ & 0.002 & -0.0044 & 0.011 & 0.0006 & 0.0016 & -0.006 & 0.0127 & 0.0006 \\
\hline & $\hat{\lambda}$ & -0.003 & 0.0095 & $6 \mathrm{E}-04$ & 0.0213 & -0.003 & 0.011 & 0.0006 & 0.0191 \\
\hline & & \multicolumn{4}{|c|}{$\alpha=2.5, \beta=2, \theta=1.9$ and $\lambda=2$} & \multicolumn{4}{|c|}{$\alpha=2.5, \beta=2.2, \theta=1.9$ and $\lambda=2$} \\
\hline \multirow[t]{4}{*}{200} & $\hat{\alpha}$ & 0.0208 & -0.0018 & 0.00357 & -0.0059 & 0.02 & -0.0004 & 0.004 & -0.005 \\
\hline & $\hat{\beta}$ & -0.00179 & 0.03489 & -0.0097 & 0.01598 & $-4 E-04$ & 0.044 & -0.012 & 0.0171 \\
\hline & $\hat{\theta}$ & 0.00357 & -0.0097 & 0.01836 & 0.00049 & 0.004 & -0.0116 & 0.019 & 0.0005 \\
\hline & $\hat{\lambda}$ & -0.0059 & 0.01598 & 0.00049 & 0.0222 & -0.005 & 0.0171 & $5 \mathrm{E}-04$ & 0.0211 \\
\hline \multirow[t]{4}{*}{300} & $\hat{\alpha}$ & 0.01369 & -0.0008 & 0.00368 & -0.00456 & 0.012 & -0.0001 & 0.003 & -0.004 \\
\hline & $\hat{\beta}$ & -0.00075 & 0.01746 & -0.00737 & 0.009022 & $-1 \mathrm{E}-04$ & 0.0366 & -0.009 & 0.0131 \\
\hline & $\hat{\theta}$ & 0.00368 & -0.0074 & 0.02498 & 0.000188 & 0.003 & -0.0091 & 0.013 & $-2 \mathrm{E}-06$ \\
\hline & $\hat{\lambda}$ & -0.00456 & 0.00902 & 0.00019 & 0.014396 & -0.004 & 0.0131 & $-2 \mathrm{E}-06$ & 0.0143 \\
\hline \multirow[t]{4}{*}{400} & $\hat{\alpha}$ & 0.00954 & -0.0003 & 0.00199 & -0.00303 & 0.009 & 0.0003 & 0.002 & -0.003 \\
\hline & $\hat{\beta}$ & -0.00033 & 0.01652 & -0.00523 & 0.00792 & 3E-04 & 0.0203 & -0.006 & 0.0084 \\
\hline & $\hat{\theta}$ & 0.00199 & -0.0052 & 0.01196 & 0.000468 & 0.002 & -0.0061 & 0.013 & 0.0005 \\
\hline & $\hat{\lambda}$ & -0.00303 & 0.00792 & 0.00047 & 0.012542 & -0.003 & 0.0084 & $5 \mathrm{E}-04$ & 0.0119 \\
\hline
\end{tabular}

Table 3: Confidence interval for model parameters at confidence level $95 \%$ using normal approximation and percentile bootstrap methods

\begin{tabular}{|c|c|c|c|c|c|c|c|c|c|}
\hline \multirow[b]{2}{*}{$\mathrm{n}$} & \multirow{2}{*}{ Parameter } & \multicolumn{2}{|c|}{ Using normal approximation } & \multicolumn{2}{|c|}{ Using percentile bootstrap } & \multicolumn{2}{|c|}{ Using normal approximation } & \multicolumn{2}{|c|}{ Using percentile bootstrap } \\
\hline & & lower & upper & lower & upper & lower & upper & lower & upper \\
\hline & & \multicolumn{4}{|c|}{$\alpha=2, \beta=1.8, \theta=1.6$ and $\lambda=1.4$} & \multicolumn{4}{|c|}{$\alpha=2, \beta=1.8, \theta=1.6$ and $\lambda=2$} \\
\hline \multirow[t]{4}{*}{200} & $\alpha$ & 1.7818 & 2.2583 & 1.7962 & 2.2546 & 1.7814 & 2.258 & 1.796 & 2.2546 \\
\hline & $\beta$ & 1.5427 & 2.1912 & 1.3236 & 2.5481 & 1.5448 & 2.193 & 1.324 & 2.5481 \\
\hline & $\theta$ & 1.4428 & 1.8756 & 0.9858 & 2.6263 & 1.4411 & 1.874 & 0.974 & 2.6264 \\
\hline & $\lambda$ & 1.1472 & 1.6744 & 1.1777 & 1.6674 & 1.6387 & 2.392 & 1.683 & 2.3821 \\
\hline \multirow{4}{*}{300} & $\alpha$ & 1.8218 & 2.212 & 1.8266 & 2.2167 & 1.8218 & 2.212 & 1.826 & 2.2167 \\
\hline & $\beta$ & 1.6111 & 2.0725 & 1.3978 & 2.3697 & 1.6112 & 2.073 & 1.398 & 2.3698 \\
\hline & $\theta$ & 1.3925 & 1.8957 & 1.085 & 2.4185 & 1.3927 & 1.896 & 1.085 & 2.4185 \\
\hline & $\lambda$ & 1.198 & 1.6101 & 1.2175 & 1.5986 & 1.7114 & 2.3 & 1.739 & 2.2837 \\
\hline
\end{tabular}


Eman A. El-Dessouky Nassef / Journal of Mathematics and Statistics 2020, Volume 16: 148.160 DOI: 10.3844/jmssp.2020.148.160

\begin{tabular}{|c|c|c|c|c|c|c|c|c|c|}
\hline \multirow[t]{5}{*}{400} & $\alpha$ & 1.8521 & 2.1731 & 1.8508 & 2.1878 & 1.8518 & 2.173 & 1.851 & 2.1878 \\
\hline & $\beta$ & 1.6072 & 2.0589 & 1.4612 & 2.2897 & 1.6082 & 2.06 & 1.461 & 2.2897 \\
\hline & $\theta$ & 1.4667 & 1.815 & 1.1469 & 2.2597 & 1.4656 & 1.814 & 1.146 & 2.2597 \\
\hline & $\lambda$ & 1.2108 & 1.6108 & 1.2551 & 1.586 & 1.7297 & 2.301 & 1.793 & 2.2657 \\
\hline & & \multicolumn{4}{|c|}{$\alpha=2, \beta=1.8, \theta=1.9$ and $\lambda=2$} & \multicolumn{4}{|c|}{$\alpha=2, \beta=2.2, \theta=1.9$ and $\lambda=2$} \\
\hline \multirow[t]{3}{*}{200} & $\alpha$ & 1.7879 & 2.2507 & 1.7993 & 2.2471 & 1.7957 & 2.24 & 1.805 & 2.2381 \\
\hline & $\beta$ & 1.5415 & 2.1884 & 1.334 & 2.5255 & 1.8869 & 2.709 & 1.612 & 3.217 \\
\hline & $\lambda$ & 1.6385 & 2.3924 & 1.6828 & 2.3817 & 1.6581 & 2.371 & 1.695 & 2.362 \\
\hline \multirow[t]{4}{*}{300} & $\alpha$ & 1.8277 & 2.205 & 1.8311 & 2.2143 & 1.8439 & 2.187 & 1.837 & 2.1999 \\
\hline & $\beta$ & 1.6093 & 2.0727 & 1.3986 & 2.3562 & 1.8854 & 2.635 & 1.691 & 2.9409 \\
\hline & $\theta$ & 1.6531 & 2.2523 & 1.2978 & 2.8666 & 1.7232 & 2.178 & 1.288 & 2.8495 \\
\hline & $\lambda$ & 1.7109 & 2.3007 & 1.7394 & 2.2835 & 1.7152 & 2.295 & 1.75 & 2.2711 \\
\hline \multirow[t]{4}{*}{400} & $\alpha$ & 1.8558 & 2.168 & 1.8536 & 2.181 & 1.8603 & 2.162 & 1.859 & 2.1754 \\
\hline & $\beta$ & 1.6081 & 2.0592 & 1.4692 & 2.2802 & 1.9709 & 2.529 & 1.773 & 2.8448 \\
\hline & $\lambda$ & 1.7293 & 2.3016 & 1.7929 & 2.2658 & 1.7436 & 2.285 & 1.801 & 2.2523 \\
\hline & & \multicolumn{4}{|c|}{$\alpha=2.5, \beta=2, \theta=1.9$ and $\lambda=2$} & \multicolumn{4}{|c|}{$\alpha=2.5, \beta=2.2, \theta=1.9$ and $\lambda=2$} \\
\hline \multirow[t]{4}{*}{200} & $\alpha$ & 2.2406 & 2.8063 & 2.2532 & 2.8041 & 2.2445 & 2.8003 & 2.256 & 2.7975 \\
\hline & $\beta$ & 1.7115 & 2.4438 & 1.4759 & 2.8487 & 1.8872 & 2.7092 & 1.612 & 3.217 \\
\hline & $\theta$ & 1.7043 & 2.2354 & 1.1604 & 3.1300 & 1.6943 & 2.2389 & 1.1448 & 3.1285 \\
\hline & $\lambda$ & 1.7180 & 2.3023 & 1.7484 & 2.9232 & 1.7252 & 2.2948 & 1.7522 & 2.285 \\
\hline \multirow[t]{4}{*}{300} & $\alpha$ & 2.2906 & 2.7493 & 2.2945 & 2.7582 & 2.3047 & 2.7336 & 2.2967 & 2.7499 \\
\hline & $\beta$ & 1.7903 & 2.3083 & 1.5475 & 2.6540 & 1.8854 & 2.6349 & 1.6908 & 2.9409 \\
\hline & $\theta$ & 1.6431 & 2.2626 & 1.2907 & 2.8505 & 1.7223 & 2.1772 & 1.2877 & 2.8497 \\
\hline & $\lambda$ & 1.7681 & 2.2384 & 1.7914 & 2.2183 & 1.7685 & 2.2375 & 1.7975 & 2.2141 \\
\hline \multirow[t]{4}{*}{400} & $\alpha$ & 2.3231 & 2.7061 & 2.3195 & 2.7205 & 2.3254 & 2.7024 & 2.3241 & 2.7192 \\
\hline & $\beta$ & 1.7879 & 2.2918 & 1.6226 & 2.5536 & 1.9708 & 2.5291 & 1.7729 & 2.8447 \\
\hline & $\theta$ & 1.7329 & 2.1616 & 1.3471 & 2.6971 & 1.7237 & 2.1658 & 1.337 & 2.701 \\
\hline & $\lambda$ & 1.7917 & 2.2307 & 1.8360 & 2.2043 & 1.7967 & 2.2251 & 1.839 & 2.1994 \\
\hline
\end{tabular}

Table 4: The expected and confidence interval for warranty cost models at confidence level $95 \%$ for two types of warranty models FRW and PRW

\begin{tabular}{|c|c|c|c|c|c|c|c|c|c|c|}
\hline \multirow[b]{2}{*}{$\mathrm{n}$} & \multicolumn{2}{|l|}{ FRW } & \multicolumn{8}{|l|}{ PRW } \\
\hline & $E\left(C N_{w f}\left(t_{w f}\right)\right.$ & $\begin{array}{l}\text { Lower- } \\
\text { normal }\end{array}$ & $\begin{array}{l}\text { Upper- } \\
\text { normal }\end{array}$ & $\begin{array}{l}\text { Lower- } \\
\text { boot }\end{array}$ & $\begin{array}{l}\text { upper- } \\
\text { boot }\end{array}$ & $E\left(C N_{w p}\left(t_{w p}\right)\right.$ & $\begin{array}{l}\text { Lower- } \\
\text { normal }\end{array}$ & $\begin{array}{l}\text { upper- } \\
\text { normal }\end{array}$ & $\begin{array}{l}\text { Lower- } \\
\text { boot }\end{array}$ & $\begin{array}{l}\text { upper- } \\
\text { boot }\end{array}$ \\
\hline \multicolumn{11}{|c|}{$\alpha=2, \beta=1.8, \theta=1.6$ and $\lambda=2$} \\
\hline 200 & 97.057 & 94.17 & 104.4 & 55.38 & 157.8 & 153.13 & 129.8 & 184.05 & 111.3 & 214.68 \\
\hline 300 & 98.846 & 94.086 & 107.3 & 63.805 & 144.9 & 155.1 & 133.6 & 182.2 & 120.44 & 201.14 \\
\hline 400 & 99.047 & 94.747 & 105.9 & 69.227 & 137.7 & 155.19 & 132.8 & 181.53 & 126.05 & 193.77 \\
\hline \multicolumn{11}{|c|}{$\alpha=2, \beta=1.8, \theta=1.9$ and $\lambda=2$} \\
\hline 200 & 82.901 & 79.384 & 90.74 & 44.551 & 140.2 & 136.86 & 115.7 & 165.09 & 97.071 & 195.29 \\
\hline 300 & 84.529 & 79.306 & 93.31 & 52.42 & 128.4 & 138.66 & 119.3 & 163.21 & 106.19 & 183.02 \\
\hline 400 & 84.71 & 80.167 & 91.69 & 56.912 & 121.9 & 138.74 & 118.3 & 162.91 & 110.79 & 176.38 \\
\hline \multicolumn{11}{|c|}{$\alpha=2, \beta=2.2, \theta=1.9$ and $\lambda=2$} \\
\hline 200 & 65.395 & 60.685 & 74.5 & 29.289 & 123.2 & 116.22 & 98.74 & 140.36 & 76.02 & 176.11 \\
\hline 300 & 67.147 & 60.648 & 77.37 & 37.035 & 111.1 & 118.23 & 102.9 & 138.26 & 85.815 & 163.36 \\
\hline \multicolumn{11}{|c|}{$\alpha=2.5, \beta=2, \theta=1.9$ and $\lambda=2$} \\
\hline 200 & 122.2132 & 120.978 & 124.73 & 78.157 & 178.86 & 195.565 & 161.285 & 239.262 & 156.787 & 251.134 \\
\hline 300 & 122.7933 & 120.836 & 128.808 & 87.917 & 167.731 & 197.467 & 166.465 & 235.481 & 166.179 & 240.108 \\
\hline 400 & 122.959 & 121.3619 & 127.289 & 92.585 & 161.235 & 197.485 & 165.760 & 234.424 & 170.240 & 233.641 \\
\hline \multicolumn{11}{|c|}{$\alpha=2.5, \beta=2.2, \theta=1.9$ and $\lambda=2$} \\
\hline 200 & 111.18 & 108.38 & 116 & 67.472 & 171.2 & 184.6 & 152.4 & 226.01 & 143.74 & 242.71 \\
\hline 300 & 113.25 & 109.9 & 120.7 & 78.172 & 158.9 & 186.8 & 158.6 & 221.64 & 154.72 & 230.14 \\
\hline 400 & 113.4 & 111.19 & 118.4 & 82.683 & 153.3 & 186.91 & 156.7 & 222 & 158.91 & 224.79 \\
\hline
\end{tabular}

\section{Conclusion}

In this study, we presented the prediction for warranty cost when our models are free rebate warranty and Pro rata rebate warranty based on partial accelerated life test with constant stress type II censoring data. We use the Maximum likelihood method to estimate the parameters of our model when 
the failure time follows Dagum distribution. We construct confidence intervals for model parameters using normal approximation and Bootstrap. From the values of RAB and MSE in six cases of parameters, we can observe that the numerical results support the theoretical findings. The expected warranty cost and its confidence intervals for two types of warranty FRW and PRW are effected with the estimated value of Dagum distribution parameters.

\section{Acknowledgment}

The author gratefully acknowledge with thanks the very thoughtful and constructive comments and suggestions of the Editor and the reviewers which resulted in much improved paper.

\section{Ethics}

The author declares that there is no conflict of interests regarding the publication of this article.

\section{References}

Abdel-Ghaly, A. A., Amin, Z. H., \& Omar, D. A. (2008). Estimation of the Burr-XII distribution for partially accelerated life tests using censored data. Model Assisted Statistics and Applications, 3(4), 317-334.

Al-Zahrani, B. (2016). Reliability test plan based on Dagum distribution. International Journal of Advanced Statistics and Probability, 4(1), 75-78.

Amberkar, S., \& Jagtap, M. M. (2014). Warranty cost modelling and analysis. International Journal of Scientific \& Engineering Research, 5(12), 40-44.

Bai, D. S., \& Chung, S. W. (1992). Optimal design of partially accelerated life tests for the exponential distribution under type-I censoring. IEEE Transactions on Reliability, 41(3), 400-406.

Bai, D. S., Chung, S. W., \& Chun, Y. R. (1993). Optimal design of partially accelerated life tests for the lognormal distribution under type I censoring. Reliability Engineering \& System Safety, 40(1), 85-92.

Blischke, W. R., \& Murthy, D. N. P. (1994). Warranty cost analysis. Marcel Dekker, Inc., New York.

Chen, Z., Zhao, T., Luo, S., \& Sun, Y. (2017). Warranty cost modeling and warranty Length Optimization under Two types of failure and combination free replacement and Pro-Rata warranty. IEEE Access, 5, 11528-11539.

Cheng, Y. F., \& Wang, F. K. (2012). Estimating the Burr XII parameters in constant-stress partially accelerated life tests under multiple censored data. Communications in Statistics-Simulation and Computation, 41(9), 1711-1727.
Dagum, C. (1977). New model of personal incomedistribution-specification and estimation. Economie appliquée, 30(3), 413-437.

Degroot, M. H., \& Goel, P. K. (2006). Bayesian estimation and optimal designs in partially accelerated life testing. Naval Res. Logist, 26, 223-235.

Díaz, V. G., Gómez, J. F., López, M., Crespo, A., \& de León, P. M. (2009). Warranty cost models State-ofArt: A practical review to the framework of warranty cost management. In ESREL (Vol. 2009, pp. 2051-2059).

Domma, F., Condino, F., \& Giordano, S. (2018). A new formulation of the Dagum distribution in terms of income inequality and poverty measures. Physica A: Statistical Mechanics and its Applications, 511, 104-126.

Domma, F., Giordano, S., \& Zenga, M. (2011). Maximum likelihood estimation in Dagum distribution with censored samples. Journal of Applied Statistics, 38(12), 2971-2985.

Domma, F., Latorre, G., \& Zenga, M. (2012). The Dagum distribution in reliability analisys. Statistica \& Applicazioni, 10(2).

Ismail, A. A. (2009). Optimum constant-stress partially accelerated life test plans with type-II censoring: the case of Weibull failure distribution. Bulletin of Statistics \& Economics,(BSE), 3(S09).

Ismail, A. A. (2014). On designing constant-stress partially accelerated life tests under time-censoring. Strength of Materials, 46(1), 132-139.

Kamal, M., Zarrin, S., \& Islam, A. U. (2013). Constant stress partially accelerated life test design for inverted Weibull distribution with type-I censoring. Algorithms Research, 2(2), 43-49.

Mittal, N. (2013). Some optimum fully and partially accelerated life testing models in reliability.

Murthy, D. N. P., \& Rodin, E. Y. (1990). A new warranty costing model. Mathematical and Computer Modelling, 13(9), 59-69.

Park, M., \& Pham, H. (2010). Warranty cost analyses using quasi-renewal processes for multicomponent systems. IEEE Transactions on Systems, Man and Cybernetics-Part A: Systems and Humans, 40(6), 1329-1340.

Rahman, A. (2007). Modelling and analysis of reliability and costs for lifetime warranty and service contract policies (Doctoral dissertation, Queensland University of Technology).

Sen, D. (1999). Accelerated life testing: concepts and models (Doctoral dissertation, Concordia University).

Wang, H. (2006). Warranty cost models considering imperfect repair and preventive maintenance. Bell Labs Technical Journal, 11(3), 147-159. 
Eman A. El-Dessouky Nassef / Journal of Mathematics and Statistics 2020, Volume 16: 148.160 DOI: 10.3844/jmssp.2020.148.160

Yang, G. (2010). Accelerated life test plans for predicting warranty cost. IEEE Transactions on Reliability, 59(4), 628-634.

Young, T. M., Perhac, D. G., Guess, F. M., \& León, R. V. (2007). Bootstrap confidence intervals for percentiles of reliability data for wood plastic composites. Forest Products Journal. Personal communication.
Zarrin, S., Kamal, M., \& Saxena, S. (2012). Estimation in constant stress partially accelerated life tests for Rayleigh distribution using type-I censoring. Reliability: Theory \& Applications, 7(4 (27)).

Zhao, X., \& Xie, M. (2017). Using accelerated life tests data to predict warranty cost under imperfect repair. Computers \& Industrial Engineering, 107, 223-234. 